\title{
Exercise training increases size of hippocampus and improves memory
}

\author{
PNAS (2011) vol. 108 | no. 7 | 3017-3022
}

\section{Natasha Sayal}

Centre for Systems Biology and Bioinformatics, Panjab University, Chandigarh, INDIA

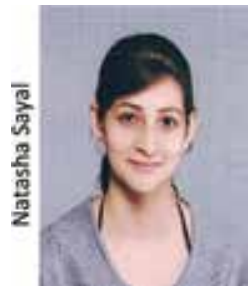

\section{Background}

Benefits of exercise are well known which includes increase in cerebral blood volume, enhancement of neural connectivity to hippocampus and influence on cognitive patterns of brain. Various studies by Kramer et al' shows exercise training largely influences the managerial control processes which includes planning, multitasking and dealing with distractions. Similarly various studies on the rodents also reported the benefits of exercise which includes enhanced memory. Study by Praag et $a^{2}$ (2005) reported the enhancement in learning, retention and hippocampal neurogenesis in aged rodents by exercise. Therefore, exercise may be an important translational approach to study neuro-cognition. Ample amount of evidence are available which shows that exercise promotes faster rate of learning and improves retention on tasks communicated by the hippocampus. Colcombe et $a^{\beta}$ have shown that gray and white matter volume in prefrontal cortex is increased by exercise training but extend to which exercise can modify the size of the hippocampus region was still unknown.

\section{Study Design}

In this study, the authors have designed a randomized controlled experiment with 120 healthy older adults distributing them equally into two exercise groups. 60 individuals (67.6 \pm 5.81 years) are subjected to aerobic exercise with 10 minutes of walking session gradually increasing to 40 minutes over a period of 7 weeks. Other 60 individuals $(65.5 \pm 5.44$ years $)$ are subjected to stretching control combining muscle toning exercise, yoga sequence and balance exercise. They have analyzed the effect of exercise on the hippocampus volume and spatial memory at two time points viz., at 6 months and one year. Hippocampal changes were analyzed by MRI and spatial memory paradigm by computerized task correlated with their blood serum levels of BDNF.

\section{Implications}

Their results clearly indicate the role of aerobic exercise in neuro-protection by the enhancement of the hippocampal volume and increase in the serum BDNF. This enhancement is further supplemented with the increase in their spatial memory. Therefore, this study suggests that starting an exercise regimen later in life is not pointless. So one year moderate intensity exercise is useful to intensify cognition or supplement brain volume.

doi : 10.5214/ans.0972.7531.220209

\section{References}

1. Kramer A F, Erickson K I. Capitalizing on cortical plasticity: influence of physical activity on cognition and brain function. Trends in cognitive sciences. 2007; 11(8): 342-348.

2. Praag van $H$, Shubert $T$, Zhao $C$. Exercise enhances learning and hippocampal neurogenesis in aged mice. J. Neurosci. 2005; 25 : 8680-8685.

3. Colcombe SJ, Aerobic exercise training increases brain volume in aging humans. J Gerontol A Biol Sci Med Sci. 2006; 61: 1166-1170. 